\title{
LA ESTELA DE RETUGENOS (K.12.1) Y EL IMPERATIVO CELTIBÉRICO
}

\author{
JESÚs ARENAS, PATRIZIA DE BERNARDO STEMPEL, \\ M CRUZ GonzÁlEZ Y JOAQUÍN GORROCHATEGUI \\ Dpt. of Archaeology, Univ. of Wales Lampeter, Dept. of Welsh, \\ Prifysgol Cymru Aberystwyth, Universidad del País Vasco, Vitoria
}

\begin{abstract}
New reading and interpretation of a Celtiberian stele from the second half of the 2 nd century BC found in Langa de Duero (Soria): retukeno esto Deltis, "of Retugenos be it the stele". The archaic inscription, itself apparently shaped as an axe, features an axe at the beginning of the word ke.l.ti.s 'stele' (*kel-ti-s) and may have been commemorating the famous Numantian

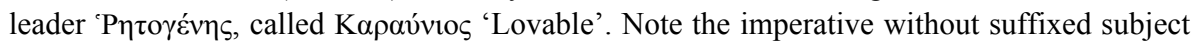
pronoun and its still preserved $-\bar{o} \#$.
\end{abstract}

El presente trabajo colectivo propone una nueva interpretación de la inscripción celtibérica sobre estela de piedra caliza procedente de Langa de Duero (Soria), cuya lectura, desde su descubrimiento en 1928, ha resultado problemática dado que uno de los signos existentes en el epígrafe era identificado como una letra ${ }^{1}$, correspondiendo, por el contrario, a un símbolo ${ }^{2}$ probablemente alusivo al estatus del personaje al que está dedicada la pieza.

${ }^{1}$ Cf. entre otros A. Tovar, FS Menéndez Pidal. Madrid, 1951, II, p. 279, U. Schmoll, Die Sprachen der vorkeltischen Indogermanen Hispaniens und das Keltiberische, Wiesbaden, 1959, p. 22 n²6, J. Untermann, GS Tovar \& Michelena. Salamanca - Vitoria, 1990, pp.354s.

${ }^{2}$ Identificado por parte de J. Arenas.

EMERITA. Revista de Lingüística y Filología Clásica (EM) — LXIX 2, 2001

pp. $307-318$ 


\section{La decoración de la estela y su contexto arqueológico (J.A.)}

La estela, que se conserva en el Museo Numantino de Soria, fue hallada entre las piedras que formaban una cerca de uso agrícola de época reciente. Carece, por lo tanto, de contexto estratigráfico, pero el entorno arqueológico del que procede y sus rasgos epigráficos permiten fecharla en la segunda mitad del siglo II a.C.:

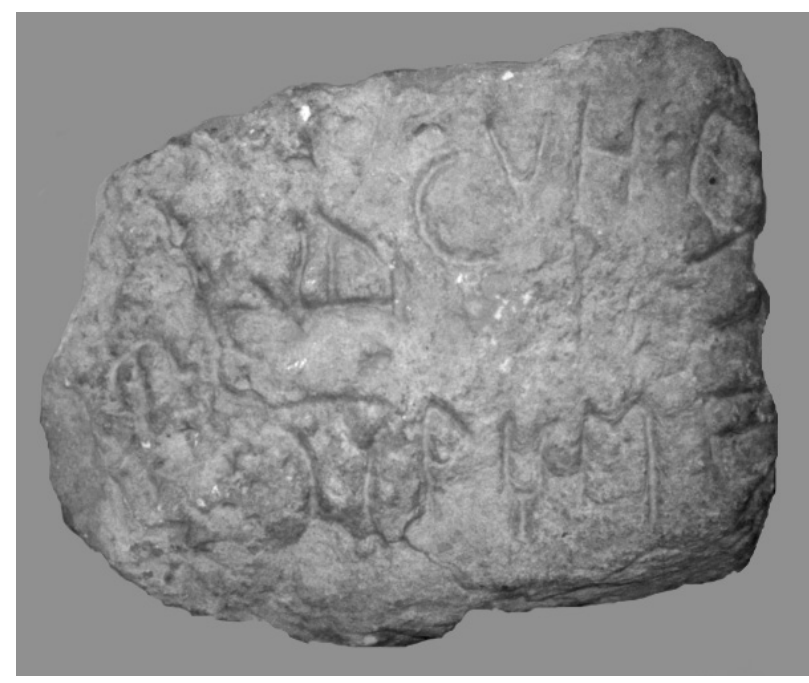

Figura 1:

Estela de Retugenos

(Conservada en el Museo

Numantino de Soria, fotografía de J. Arenas con autorización de la Consejería de Cultura de la Junta de Comunidades de Castilla y León).

El hallazgo del epígrafe se inscribe en un amplio programa de excavaciones que B. Taracena inició en 1928 en un paraje genéricamente conocido como el Cerro del Moro $^{3}$, que es en realidad una extensa zona arqueológica constituida por varios yacimientos de distinto tipo y cronología. De todos ellos, el que merece nuestra atención es el conocido como Las Quintanas, donde fue localizado un conjunto de construcciones de época celtibérica que pueden ser fechadas, a partir de su contenido mobiliar, a lo largo del siglo II y parte del I a.C.

La posibilidad de vincular cronológicamente nuestra inscripción a cualquiera de los otros yacimientos inspeccionados es muy escasa, pues según la memoria de excavaciones, además de en Las Quintanas, se actuó en asentamientos datables, bien en la Primera Edad del Hierro, bien en época medie-

3 B. Taracena Aguirre, Excavaciones en las provincias de Soria y Logroño, Junta Superior de Excavaciones y Antigüedades, Memoria $n^{\circ}$ general 103 (Madrid, 1929), p. 50. 
val. Las Quintanas, sin embargo, ofrecen una cultura material característica de la segunda mitad del siglo II a.C., ya que, a excepción de un ánfora vinaria de tipología itálica (por otra parte presentes en esta zona de la Meseta ya en la primera mitad del siglo II a.C.) carece de los productos de importación (como la cerámica Campaniense A y B) ${ }^{4} \mathrm{o}$ indígenas (como la cerámica polícroma de estilo numantino) que se generalizan en la zona a partir de la caída de Numancia en el 133 a.C.

Desde un punto de vista estrictamente arqueológico cabe centrar nuestra atención en un detalle existente en el texto que ha pasado hasta el momento desapercibido a todos los especialistas que han estudiado la pieza: en el extremo izquierdo de la línea inferior existe un carácter que rebasa en más de dos centímetros el tamaño de los caracteres epigráficos a los que acompaña ${ }^{5}$. Responde a la forma de carrete en posición vertical y su lado derecho ha sido aprovechado para representar la primera de las letras de la línea. Su identificación era difícil, ya que su extremo inferior está prácticamente perdido a causa de la erosión del soporte, conservándose tan sólo la zona más profunda del surco inciso.

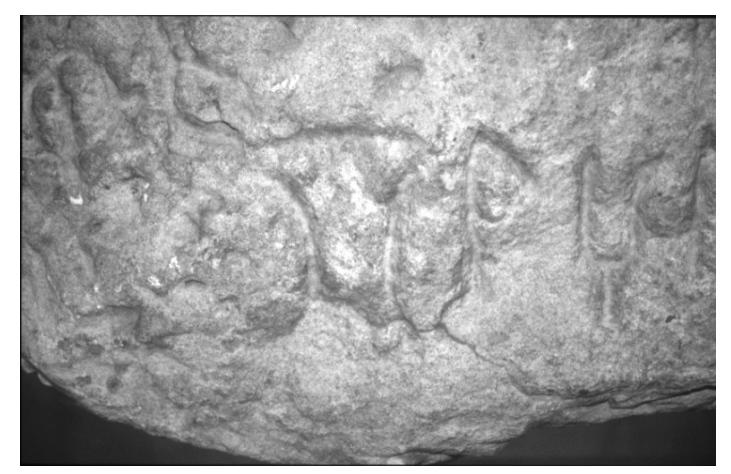

Figura 2:

Detalle del símbolo existente en el tramo inferior del texto.

La interpretación de este signo es problemática, pero no sería arriesgado pensar que se trate de una doble hacha o labris. Lo cierto es que este motivo

4 E. Sanmartí y J. Principal, «Las cerámicas de importación itálicas e ibéricas procedentes de los campamentos numantinos», Revista d'Arqueologia de Ponent 7, 1997, pp. 3575; $M^{a}$ L. Cerdeño, E. Sanmartí y R. García Huerta, «Las relaciones comerciales de los Celtíberos» en F. Burillo Mozota (coord.), IV Simposio sobre Celtíberos: Economía (Zaragoza 1999), pp. 263-299 (v. especialmente pp. 290ss.).

${ }^{5} 10 \mathrm{~cm}$. vs. 8 u 8,5cm., cf. J. Untermann, Monumenta linguarum Hispanicarum, Band IV unter Mitwirkung von D. Wodtko (Wiesbaden, 1997), p. 680. 
aparece en otras estelas prerromanas de la península Ibérica ${ }^{6} \mathrm{y}$, de forma mucho más habitual, en los repertorios ornamentales de la cerámica de la Edad del Hierro peninsular.

Efectivamente, elementos similares forman parte de los esquemas decorativos de la cerámica de época ibérica en el valle del Ebro y el Bajo Aragón ${ }^{7}$, pero es en la cerámica pintada celtibérica de los siglos II y I a.C. donde su presencia es, no sólo más reiterada, sino también significativa, por la carga simbólica que conlleva. Algunas de las piezas recuperadas en yacimientos como Los Castellares de Herrera de los Navarros en Zaragoza ${ }^{8}$, Numancia ${ }^{9}$, Izana ${ }^{10}$ y Las Quintanas de Langa de Duero ${ }^{11}$ en Soria muestran la reiterada asociación del labris y una o varias esvásticas:

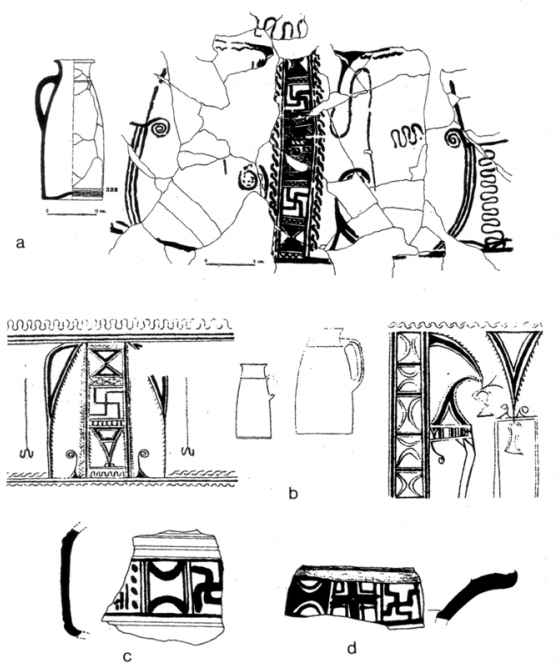

Figura 3:

Diversos ejemplos de la asociación labris-tetraskelion presentes en la cerámica celtibérica:

a) Numancia (Soria).

b) Izana (Soria).

c) Las Quintanas (Langa de Duero, Soria).

d) Los Castellares (Herrera de los Navarros, Zaragoza).

6 J.E. Cirlot, Diccionario de Símbolos (Madrid, 1997; $1^{\text {a }}$ ed., 1958), s.v. hacha.

7 M. Beltrán Lloris, Arqueología e Historia de las ciudades antiguas del Cabezo de Alcalá de Azaila (Teruel) (Zaragoza, 1976), p. 263.

8 F. Burillo Mozota, El poblado de época ibérica y yacimiento medieval: "Los Castellares" (Herrera de los Navarros, Zaragoza) - I (Zaragoza, 1983), p. 85.

9 F. Romero Carnicero, Las cerámicas polícromas de Numancia (Valladolid, 1977), figs. 26 y 37.

10 B. Taracena Aguirre, Excavaciones en las provincias de Soria y Logroño. Junta Superior de Excavaciones y Antigüedades, Memoria $n^{\circ}$ general 86 (Madrid, 1927), lámina VII.

11 Ob. cit. nota 3: lámina VIII. 
Según Cirlot $^{12}$, la doble hacha es un ideograma de amplia dispersión geográfica que suele vincularse a la idea de poder y prestigio. Por su parte, la esvástica o tetraskelion, a la que en ocasiones se encuentra asociado, representa, sobre todo en la Edad del Hierro, al dios supremo, la rueda solar, etc. Un binomio simbólico que encaja bien con la nueva lectura del epígrafe que se propone en este trabajo: un ideograma y un epígrafe asociados para ensalzar la gloria de un personaje.

Este recurso no es desconocido en el mundo prerromano peninsular, ya que es relativamente frecuente encontrar imágenes simbólicas utilizadas para ensalzar la gloria de un personaje o círculo parental. Son bien conocidos casos como el de los vadinienses en el norte peninsular, que se asocian a la imagen del caballo ${ }^{13}$, o las estelas anepígrafas bajoaragonesas, en las que se representan lanzas que, en número variable, hacen referencia a la gloria del individuo al que estuvieron vinculadas ${ }^{14}$. En ámbitos célticos extrapeninsulares encontramos un notable ejemplo en la estela de San Bernardino de Briona $\left(\right.$ Novara, Italia) ${ }^{15}$, donde un epitafio queda asociado a cuatro ruedas de ocho radios (representativas de un carro) con las que se pretende significar el estatus de los personajes que se enterraron junto a ella ${ }^{16}$. También se discute como fenómeno de origen céltico el formulario sub ascia dedicauit, frecuente en epitafios latinos de época imperial en la Cisalpina y en la Lugdunensis ${ }^{17}$.

12 Ob. cit. nota 4.

${ }^{13}$ Cf. González Rodríguez, Los Astures y los Cántabros Vadinienses, Anejos de Veleia S.M. 10, Vitoria-Gasteiz, 1997, pp. 98 con bibliografía y 113-115.

${ }^{14} \mathrm{Cf}$. F. Marco Simón, «Nuevas estelas ibéricas de Alcañiz», Pyrenae 12, 1976, pp. 84ss.

${ }^{15}=\mathrm{n}^{\circ} \mathrm{E}-1$ de M. Lejeune, Recueil des Inscriptions Gauloises, II/1: Textes gallo-étrusques. Textes gallo-latins sur pierre, París, 1988, pp. 11-24. Una interpretación modificada (con 2 sepultados y 1 oferente de la estela frente a los 5 sepultados de la interpretación tradicional) es la propuesta por De Bernardo Stempel, «From Indo-European to the Individual Celtic Languages», FS G. Mac Eoin, Dublín, 2001, § 7.4.

${ }^{16}$ Cf. E.P. Hamp, «Varia XLII: Briona @ ๑ @ ๑ », ÉC 27, 1990, p. 181.

${ }^{17} \mathrm{M}^{\mathrm{a}}$ G. Arrigoni Bertini, «L'ascia in Cisalpina. Considerazioni», XI Congresso Internazionale di Epigrafia Greca e Latina, Roma, 1999, pp. 629-637. - Nótese que de la treintena de inscripciones de la Galia transalpina seleccionadas por la Epigraphische Datenbank de Heidelberg (agradecemos a la Dra. Elena Torregaray su ayuda informática) casi un tercio son también conmemorativas de los dedicantes mismos, cf. p.e. HD027951, donde Toutedo y Atevrita parentes miserrimi de linaje céltico et sibi uiii posuerunt et sub ascia dedikauerunt, o, aún más claramente, la HD012273, donde el maritus et sibi uiu(u)s posterisque suis fecit et sub ascia dedicauit. 


\section{La lectura del texto (J.G.)}

El texto, en escritura celtibérica occidental, describe un recorrido triangular a través de tres segmentos diferenciados: el signo $R$-, a partir del cual se bifurcan los segmentos superior e inferior, corresponde a la primera letra del nombre personal y constituye el vértice inicial del texto. El segmento superior o hipotenusa del triángulo contiene el resto del nombre, -eTuKeno, y queda nítidamente separado por tres puntos del siguiente segmento vertical, en el que se puede leer esTo con orientación destrorsa. Por último, el inicio del tercer e inferior tramo de texto está marcado por un elemento simbólico (probablemente una doble hacha, $\mathrm{cf.} \S 1$ ) que lo separa del vértice izquierdo formado por el carácter $r$ antes citado. El signo $\square$ tiene también otras funciones, ya que, además de poseer un carácter simbólico, su trazado cóncavo derecho sirve para expresar el silabograma $\langle\mathrm{Ke}>$ (de forma parecida a la del tipo $\mathrm{n}^{\circ} 4$ de $M L H$ que se encuentra en Gruissan y Luzaga), después del cual se lee -lTis. El vértice inferior derecho no tiene ninguna marca especial, sino el hecho de que en él se enfrentan las dos líneas de lectura destrorsas de esta inscripción.

El trazado pseudotriangular del texto no se parece al de los demás epígrafes celtibéricos conocidos, quizá pueda responder a una intencionalidad difícil de determinar por el momento ${ }^{18}$, ya que no es la morfología del soporte la que lo justifica:

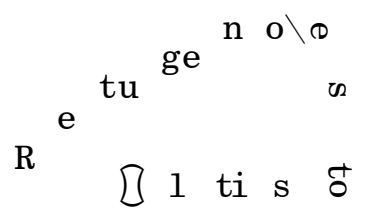

Los signos están profundamente incisos en la superficie rugosa de la piedra, lo que no impide la lectura. Solamente los tres últimos signos del texto del lado derecho, que leemos e.s.To, carecen de sus partes superiores por haber desaparecido a causa del desgaste del borde de la piedra. Pero el resto del trazado de los signos obliga con seguridad a proponer la lectura da-

\footnotetext{
${ }^{18}$ Nos preguntamos, con toda la cautela que se requiere en estos casos, si el desarrollo del texto (como ya se ha comentado, de forma aproximadamente triangular) no podría ser un refuerzo del mensaje simbólico del labris descrito.
} 
da, como bien indica Untermann en su edición del epígrafe ${ }^{19}$. El texto, como finalmente se puede leer, es, por tanto,

$$
\text { r.e.Tu.Ke.n.o } \backslash \text { e.s.To } \mid \square(\mathrm{Ke}) \text {.1.Ti.s }
$$

donde el símbolo interrelacionado con el comienzo de la última palabra se puede también entender como resumen iconográfico del texto.

\section{Interpretación del texto y comentario gramatical (P.d.B.St.)}

Hasta ahora la única palabra considerada clara de este texto que «parece sepulcral ${ }^{20}$ era r.e.Tu.Ke.n.o, es decir el nombre celta Rectugenos ${ }^{21}$ en la forma de genitivo singular celtibérico en $-o^{22}$, Retugeno, y con el desarrollo fonético $* k t>t$, también típico del celtibérico.

El Determinatum o Bezugswort del genitivo inicial está en el (Ke).l.Ti.s final del texto ${ }^{23}$ : análogamente respecto al cib. gen-ti-s 'generación $>$ hijo' ${ }^{24}$ podemos pensar en un nomen actionis en -ti- de la raíz kel- 'elevarse', es decir, en un kel-ti-s con el sentido de 'elevación > estela'.

La palabra intermedia ha sido recientemente considerada, por su vocal final $-o$, como otra forma de genitivo singular de nombre individual, aunque todavía sin posibilidad de contrastación con otros ejemplos ${ }^{25}$. Este juicio ha sido emitido a pesar de la rareza de una fórmula onomástica articulada en dos nombres individuales en genitivo singular precediendo a un nombre individual en nominativo ${ }^{26}$. Según nuestra visión, y evitando los prejuicios

\footnotetext{
${ }^{19} \mathrm{Ob}$. cit. nota 5.

${ }^{20} \mathrm{Cf}$. J. de Hoz, «La epigrafía celtibérica», Reunión sobre epigrafia hispánica de época romano-republicana, Zaragoza, 1983-86, p. 62.

${ }^{21}$ Cf. K.H. Schmidt, Die Komposition in gallischen Personennamen, Tubinga, 1957, p. 257.

${ }^{22}$ Considerado un arcaísmo por De Bernardo Stempel, «From Indo-European to the Individual Celtic Languages» (ob. cit. nota 15), § 1.1.

${ }^{23}$ Para Untermann todavía sin identificar («ohne Anschluß»: MLH IV, p. 681).

${ }^{24} \mathrm{Cf}$. Gorrochategui, «El celtibérico, dialecto arcaico celta», EMERITA 42, 1994, p. 311.

${ }^{25}$ Untermann $M L H$ IV, p. 681: «wohl ebenfalls G.Sg., bleibt ohne Anschluß: zweiter I[ndividual]N[ame]?».

${ }^{26}$ Las inscripciones funerarias que tenemos (una decena, la mitad fragmentarias) responden a otras fórmulas: unos ejemplos son la K.13.1 con Kaabaarinos, es decir, con el nominativo singular masculino de nombre individual *Kambarinos 'Cambarinos' (cf. el genitivo plural masculino de nombre de agrupación familiar Kambarokum en K.5.2) y la K.16.1
} 
mantenidos por algunos investigadores ${ }^{27}$, esto sería una forma arcaica del imperativo es-tō correspondiente al celtibérico clásico $* * e s t u^{28}$.

La inscripción, correspondiente a un originario *Rektugenō(d) estō(d) keltis, significaría por lo tanto "de Re(c)tugenos sea estela" y tendría la estructura GenSingMasc(Nombre individual) + Verbo $\left(3^{a}\right.$ SingImperat $\left.{ }^{29}\right)+$ NomSingFem/Masc(Nombre común). La posición aparentemente intermedia del verbo es en realidad ejemplo de orden de palabras VS, marcado con respecto al orden con verbo final típico del celtibérico ${ }^{30}$ y que se adapta bien a

de ca. 100 a. de C. con Tirtanos Abulokum LetonTunos Ke() BeliKios (*Tritanos Ab(u)lokōm Letondunos ge(ntis) Bel(i)k/gyos) "Tritanos de los $\mathrm{Ab}(\mathrm{u})$ loci, hijo de Letondo, ciudadano Belg-", o sea con una estructura de NomSingMasc (Nombre individual) + GenPlurMasc (Nombre de agrupación familiar) $+\{$ GenSingMasc (Nombre individual) + NomSingMasc ('hijo')\} + NomSingMasc (Étnico).

${ }^{27}$ Esta postura podría resumirse en la no aceptación de una $\bar{o}$ arcaica en celtibérico.

${ }^{28}$ Recuérdese que la existencia de formas arcaicas de imperativo en - $o \#$ fue postulada por F.R. Adrados («Aportaciones a la interpretación del bronce de Botorrita», Actas del I coloquio sobre lenguas y culturas prerromanas de la Península Ibérica (Salamanca 1974), Salamanca, 1976, pp. 25-47, y en part. 40; idem, «The Celtiberic verb», Kurytowicz memorial volume, Part one, Cracovia, 1995, pp. 447-453, y en part. 448 y 452) y - más sistemáticamente - por F. Villar («Tratamiento de -ō en sílaba final: algunas posibles formas de imperativo en celtibérico», Veleia 6/1989, pp. 199-205 y en part. 202s.) a fin de explicar el neito de Botorrita IA6, interpretado por ellos como "no vaya" (ne-ito).

${ }^{29}$ Frases con imperativo de futuro en inscripciones privadas son conocidas también en el ámbito itálico, cf. el Ni uiolato de la inscripción n ${ }^{\circ} 938$ de la colección de A. Degrassi, Inscriptiones Latinae liberae rei publicae, Florencia, 1972 ( $1^{\text {a }}$ ed.1963), frente al Nolei uiolare de la ${ }^{\circ} 937$ o la inscripción sobre canalón no 2491.153: liber-esto del corpus de R.G. Collingwood \& R.P.Wright, The Roman Inscriptions of Britain, II/5 (ed. por S.S. Frere \& R.S.O. Tomlin, Oxford, 1993). - Otros casos interesantes son algunas estelas en las que el texto encierra la intencionalidad de "para XY sea esta estela"; p.e. la inscripción picena meridional de Penna Sant'Andrea ( $=\mathrm{n}^{\circ}$ TE.5 de A. Marinetti, Le iscrizioni sudpicene: I testi, Florencia, 1985, pp. 117-130 y 215-217), cuyas últimas líneas han sido interpretadas por I.-J. Adiego Lajara, Protosabelio, osco-umbro, sudpiceno, Barcelona, 1992, p. 33, como: "Se alza [praistait < * pray-stāyett] ... como regalo [meitims : gót. maipms] del trebegio- del pueblo sabino [Safinas tutas $<*^{*}$ sab $^{h}$ inās towtās] para Tito Póstumo [Titúi Posmúi < *Titōy Postmōy] esta estela [praistakla sa $<$ *pray-stātlā sā]". En ámbitos célticos recordaremos, aunque elípticas de forma verbal, las estelas lepónticas del tipo pala (M. Lejeune, Lepontica, París 1971, pp. 80-87) como la de Davesco: Slaniai VerKalai pala : Pivonei TeKialui pala 'estela para Slania hija de Vergos (y) estela para Bivón hijo de Dengios'.

${ }^{30}$ Cf. K.H. Schmidt, «Der Beitrag der keltiberischen Inschrift von Botorrita zur Rekonstruktion der protokeltischen Syntax», Word 28/1-2, 1972, pp. 51-62 y en part. 53ss. 
una frase imperativa ${ }^{31}$.

En el ámbito del corpus celtibérico podemos comparar el simple y arcaico imperativo estō con formas como datuz $\left(<*\right.$ datus $\# \leftarrow\left\{{ }^{*}\right.$ datu $\left[<* d^{(h)} 2 t \overline{t o} d\right]$ $\left.\left.+{ }^{\text {nom.sg. }} \operatorname{sos}\right\}\right)$ u oisatuz $\left(<*_{\text {opi-sato }}(d)+s\right)^{32}$, es decir, con formas de imperativo en $-t \bar{o}(d)>-t u \#$ ampliadas por medio del pronombre sujeto de tercera persona singular, sos, en su alomorfo enclítico $-s$, «siguiendo una propuesta de Fleuriot [ÉC 18/1981, p. 91] perfectamente congruente con usos aglutinativos verbo-pronombre bien conocidos entre las lenguas celtas» ${ }^{33}$. La alternancia entre formas con pronombre enclítico de sujeto y sin él (que recuerda vagamente la alternancia del indicativo galo entre el tipo delgu y el tipo con nota augens pissiiu-mi) se justifica perfectamente por el contexto sintáctico celtibérico en el cual los dos tipos se encuentran ${ }^{34}$.

Con respecto a la vocal $-\bar{o} \#$ de estō, todavía no oscurecida en $-u \#$ en final de palabra, tenemos aquí, como en los nominativos plurales de etnónimos (cib.arc. ArKailikos [A.62], Ekualakos [A.63] ${ }^{35}$, SeKisamos [A.69] vs. cib. clás. Usamuz [A.72], Karaluz [A.65]), en los respectivos genitivos plurales en leyendas monetales ${ }^{36}$ (cib.arc. SeKaizakom [A.78], Kontebakom Karbi

\footnotetext{
${ }^{31}$ Cf., p.e., C. F. Justus, «Dislocated imperatives in the Indo-European prayer», Word 44, 1993, pp. 273-294, y en part. 276 y 281 ss. De hecho, este orden OVS es el mismo que se encuentra en otras frases imperativas celtibéricas como dekametinas datu-z en Botorrita I (v. más adelante)

${ }^{32} \mathrm{Y} d \bar{l}-(e) n$-bituz, usa-bituz, bisetuz, todas con sonorización de la sibilante sorda originaria en final absoluta.

${ }^{33}$ F. Villar 1989, ob. cit. nota 28, p. 203. Cf. también J. de Hoz, «La epigrafía celtibérica», ob. cit. nota 20, pp. 57 y 88, J.F. Eska, «The Verbal Desinence -Tus in the HispanoCeltic Inscription of Botorrita», ZCP 43, 1989, pp. 214-22, y más recientemente De Bernardo Stempel, «From Indo-European to the Individual Celtic Languages», ob. cit. nota 15, § 7.3.2 con paralelos italianos.

${ }^{34} \mathrm{Cf}$. ya Villar 1989, ob. cit nota 28: «Naturalmente, la presencia y ulterior aglutinación de * so no ha lugar cuando el mandato o prohibición expresados por el imperativo no se refiere a un individuo concreto previamente citado» (p. 204).

${ }^{35}$ V. ahora De Bernardo Stempel, «Grafemica e fonologia del celtiberico: 1. Nuovi dati sulle vocali mute», Religión, lengua y cultura prerromanas de Hispania (ed. por F. Villar y $M^{\mathrm{a}}$. P. Fernández Álvarez, Salamanca 2001), p. 322, sobre la variante abreviada /ekwalak ${ }^{\mathrm{u}}$, escrita $<\underline{\text { E. ku. a. } 1}$$$
\text { a. } \mathrm{ku}>\text {. }
$$

${ }^{36}$ Nótese que los dos tipos de leyendas celtibéricas con nominativo plural y con genitivo plural del etnónimo corresponden exactamente a los dos tipos galos, es decir el de la Galia
} 
kom [A.75], BeliKiom [A.47] vs. cib. clás. Titum [A.92], MeTuainum [A. $84]^{37}$ ) y en otros hallazgos esporádicos (como quizá kaltaikikom en K.23.2 o eventualmente arebasikom en K.9.5), otro testimonio de la fase fonética arcaica del celtibérico donde la $\bar{o}$ final todavía no se había convertido en $u^{38}$.

\section{Posibilidad de una interpretación histórica (M.C.G.)}

La identificación del lugar del hallazgo del epígrafe, el yacimiento arqueológico situado en la ladera Norte del elevado cerro llamado Cuesta del Moro de Langa de Duero, con la ciudad arévaca de Segontia Langa citada en las fuentes escritas ${ }^{39}$ y cuya cronología abarca del s. II a.C. a mediados del s. I d.C. (cf. § 1) junto con los rasgos lingüísticamente arcaicos del texto nos llevan a proponer una datación temprana para esta estela, que podría fecharse en la segunda mitad del s. II a.C.

Si a la temprana cronología sumamos la decoración del monumento (cf. § 1) y el estilo del texto de la inscripción (cf. § 3) se puede pensar que la

Cis- y Transalpina con nominativo plural (cf. las monedas lepónticas con riKoi, las galas con Eburovices, Perrucori, Senones, Velioca $\Theta i$ y las británicas antiguas con Iceni) frente al tipo con genitivo plural de la más helenizada Narbonensis (cf. las leyendas galogriegas $\Gamma \lambda \alpha v \imath \kappa \omega v$,

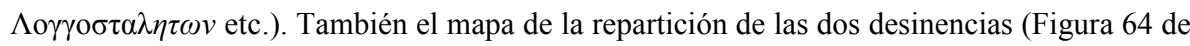
F. Burillo, Los celtíberos. Etnias y estados, Barcelona, 1998) parece confirmar la mayor cercanía del tipo en -kōm al ámbito de influencia de los modelos griegos.

${ }^{37}$ Este se leerá /Medwainum/, desde un originario celta *medw-an-yōm.

${ }^{38} \mathrm{Cf}$. De Bernardo Stempel, «Probleme der relativen Chronologie: nochmals zu idg. * ${ }^{\circ} \mathrm{im}$ Keltischen», Akten des ersten Symposiums deutschsprachiger Keltologen, Gosen 1992, ed. por M. Rockel y St. Zimmer, Tubinga, 1993, pp. 37-56 y en part. p. 40.

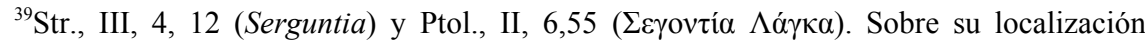
véanse entre otros: C. García Merino, Población y poblamiento en la Hispania romana. El conuentus Cluniensis, Valladolid 1975, pp. 299-300: A. Tovar, Iberische Landeskunde. T. 3, Tarraconensis, Baden-Baden 1989, pp. 347-348: Tabula Imperii Romani (K-30), Madrid 1993, pp. 208-209; M. Ramírez Sánchez, «Los núcleos de población de las comunidades indígenas del Alto Duero a través de las fuentes literarias y arqueológicas», Vegueta 3, 1997 1998, pp. 65-87 (pp. 75-76) y en último lugar F. Burillo, ob. cit. nota 36, p. 188 donde se puede encontrar también información relativa a la ceca indígena de SeKoTiaz LaKaz (sobre su etimología céltica cf. De Bernardo Stempel, Die Vertretung der indogermanischen liquiden und nasalen Sonanten im Keltischen, Innsbruck 1987 (IBS 54), p. 165 s.v. laCas y J.L. García Alonso, "On the Celticity of the Duero Plateau: Place-Names in Ptolemy», Ptolemy: Towards a linguistic atlas of the earliest Celtic place-names of Europe, ed. por D.N. Parsons \& P. Sims-Williams, Aberystwyth, 2000, pp. 37s.). 
persona de Retugenos, a cuya memoria va dedicada la estela, como aquellos otros personajes a los que van destinadas las estelas anepígrafas de tradición indígena del valle medio del Ebro ${ }^{40}$, debió haber jugado un papel importante en el seno de la sociedad celtibérica y pudo haber sido un miembro destacado de la élite indígena. Esta consideración se ve reforzada por la ausencia de la fórmula onomástica indígena propia de los celtíberos (del tipo mencionado en la nota 26), lo que nos inclina a pensar que en la época en la que se grabó la estela Retugenos era lo suficientemente conocido para que sólo su nombre individual sirviera para identificarlo ${ }^{41}$.

Por todo ello, se puede apuntar la posibilidad de una identificación entre

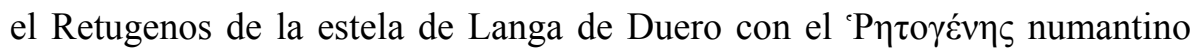

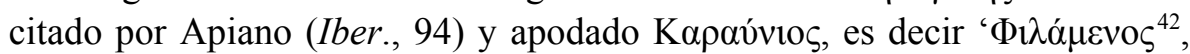

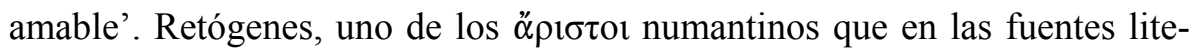
rarias son identificados únicamente por su nombre personal y, en el caso que nos ocupa, por su apodo, se había distinguido por protagonizar una noble gesta que más que el éxito personal persigue la salvación de su comunidad, Numantia, en unos momentos en los que peligra su existencia ${ }^{43}$. En consecuencia, no resulta del todo improbable que la estela aquí estudiada recuerde una hazaña y un valiente y "amable" personaje histórico que en el año 133 a.

\footnotetext{
${ }^{40} \mathrm{~F}$. Marco Simón, Las estelas decoradas de los conventos cesaraugustano y cluniense, Zaragoza 1978; F. Beltrán, «La escritura en la frontera. Inscripciones y cultura epigráfica en el valle medio del Ebro», Roma y el nacimiento de la cultura epigráfica en Occidente, Zaragoza 1995, pp. 169-195 (pp. 177-178).

${ }^{41}$ A diferencia de lo que sucede en el caso de otros miembros de la élite indígena celtibérica: los Pi.n.Ti.s de Botorrita 1 (sobre la interpretación del término cf. ÉC 32/1996) y los magistrados de Botorrita 2 o en el de los individuos documentados en Botorrita 3.

${ }^{42}$ Sobre la interpretación de la epiclesis Kapav́vios como procedente de un más antiguo celta *kara-mno-s (atestiguado en contexto venético) y su comparación semántica con el

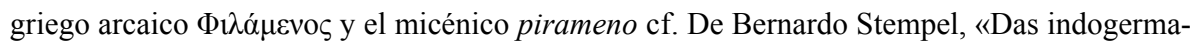
nische $m(V)$ no-Verbaladjektiv im Keltischen», Indogermanica et Caucasica: FS K. H. Schmidt, ed. por R. Bielmeier y R. Stempel con R. Lanszweert, Berlín y Nueva York 1994, pp. 281-305, en part. 287. Nótese en el apodo el mismo desarrollo fonético *-mn- $>/ \mu \mathrm{n} />$ un- esperado también por el celtibérico.

${ }^{43}$ Sobre las características de la sociedad celtíbera y, en especial, la nobilitas y las bases de su poder así como sobre su código ético cf. P. Ciprés, Guerra y sociedad en la Hispania indoeuropea, Anejos de Veleia S.M. 3, Vitoria-Gasteiz, 1993, en especial pp. 114 135.
} 
C. no dudó en arriesgar su vida junto con la de cinco pí̉oı y cabalgar "hacia las ciudades de los arévacos con ramas de olivo de suplicantes, solicitando su ayuda para los numantinos en virtud de los lazos de sangre que unían a ambos pueblos" 44 .

Una de estas ciudades arévacas - de las que lamentablemente sólo Lutia aparece mencionada en este párrafo por Apiano - pudo haber sido la que la historiografía moderna identifica con la ceca de SeKoTiaz LaKaz y la Serguntia citada por Estrabón (Langa de Duero), que habría erigido esta estela en recuerdo del valiente numantino y destacado miembro de la élite indígena que tuvo una muerte de acuerdo con su rango y dignidad ${ }^{45}$.

\footnotetext{
${ }^{44}$ App., Iber., 94. Traducción de A. Bernabé en la «Biblioteca Clásica de Gredos». Al mismo personaje se refiere presumiblemente también Floro (I 34, 11).

${ }^{45}$ Val. Max. III 2, ext. 7 informa del suicidio de Retógenes junto con un grupo de guerreros. Sobre este rito y su significado cf. P. Ciprés, ob. cit. nota 43, p. 133.
} 